\title{
Supplementary Material: \\ Molecular dynamics simulations of the 30S ribosomal subunit reveal a preferred tetracycline binding site
}

\author{
Alexey Aleksandrov and Thomas Simonson \\ Laboratoire de Biochimie (CNRS UMR7654), Department of Biology, Ecole \\ Polytechnique, 91128 Palaiseau, France.
}

\section{Full reference $16 a$}

Mackerell, A., Bashford, D., Bellott, M., Dunbrack, R., Evanseck, J., Field, M., Fischer, S., Gao, J., Guo, H., Ha, S., Joseph, D., Kuchnir, L., Kuczera, K., Lau, F., Mattos, C., Michnick, S., Ngo, T., Nguyen, D., Prodhom, B., Reiher, W., Roux, B., Smith, J., Stote, R., Straub, J., Watanabe, M., Wiorkiewicz-Kuczera, J., Yin, D., and Karplus, M. An all-atom empirical potential for molecular modelling and dynamics study of proteins. J. Phys. Chem. B 102 (1998), 3586-3616.

\section{Computational methods: Molecular dynamics (MD) simula- tions}

The crystal structure of the Thermus thermophilus 30S ribosomal subunit, complexed with tetracycline (Tc), was taken from the Protein Data Bank (PDB), entry 1HNW [1]. The complexes with minocycline (Mc) and doxycycline (Dc) were built by superimposing Mc or Dc on Tc in the $1 \mathrm{HNW}$ structure. MD simulations were performed for the primary site (TET1) and the secondary site (TET5; only for Tc) and included RNA and protein residues within a $26 \AA$ sphere, centered on the $\mathrm{C} 22$ atom of Tc (or Mc or Dc) in the primary site, or on the C6 atom of Tc in the secondary site. Notice that the sphere center does not play any special role in the model, so the precise choice is not important. Sensitivity to the position of the model boundary, on the other hand, is discussed below. Hydrogens were constructed with ideal stereochemistry. A $29 \AA$ sphere of water was overlaid and waters that overlapped protein/RNA, crystal ions, $\mathrm{Tc}$, or $\mathrm{Mg}^{2+}$ were removed. To zero the total charge and simulate ionic strength explicitly, in addition to crystal ions, 24 chloride ions and 59 sodium ions were added to the models of the primary site, and 20 chloride ions and 76 sodium ions were added to the models of the secondary site. The ions were added one by one, choosing the water 
molecule that experienced the lowest (respectively, the highest) electrostatic potential and replacing that water by a sodium (respectively, a chloride) [2]. RNA/protein atoms between $20 \AA$ and $26 \AA$ from the initial sphere's center were harmonically restrained to their Xray positions. Simulations were performed with the SSBP solvent model $[2,3]$, which treats the region outside the $26 \AA$ sphere as a uniform dielectric continuum, with a dielectric constant of 80 . This is reasonable, since most of the outer region is water. Indeed, if a parallelepiped is adjusted to fit the $30 \mathrm{~S}$ particle, its inner volume is about $91 \%$ water. Newtonian dynamics were used for the innermost region, within $20 \AA$ of the sphere's center; Langevin dynamics were used for the outer part of the sphere, referred to as the buffer region. We used a two femtosecond timestep and a bath temperature of $292 \mathrm{~K}$. The CHARMM22 force field was used for the protein [4]. The TIP3P model was used for water [5]. Electrostatic interactions between atoms within the $26 \AA$ sphere were computed without any cutoff, using a multipole approximation for distant groups [6]. Tetracycline was described with the force field developed previously [7]. The Mc and Dc force fields were developed in the same way and will be described elsewhere. The Mc and Dc force fields yield good agreement for the Tc/Mc and Tc/Dc binding free energy differences, computed with alchemical, MD free energy simulations (not to be confused with Poisson-Boltzmann free energy calculations; see below) [8, 9]. Calculations were performed with the CHARMM program [10]. Including the two binding sites, the different ligands and Tc forms, and the alchemical MD free energy simulations (see below), we performed a total of more than 150 ns of dynamics.

\section{Computational methods: $\mathrm{Mg}^{2+}$ binding free energies}

To establish that $\mathrm{Mg}^{2+}$ is bound in the TET1 and TET5 sites in the absence of Tc or Mc, we employed a more rigorous, well-established free energy simulation method; see $[11,12]$ for reviews. MD simulations were performed with the setup described above, without Tc. Water (within the $26 \AA$-radius sphere) was described explicitly, in contrast to the dielectric continuum treatment above. During the simulations, the $\mathrm{Mg}^{2+}$ cation is gradually deleted. The same procedure is performed for $\mathrm{Mg}^{2+}$ in a sphere of pure water. The free energy change is obtained from a "thermodynamic integration" formula $[8,12]$. Taking the difference between the free energies computed in the ribosome complex and in pure water yields the binding free energy [13]; Table 1. For more details, see a recent application of the same method to an enzyme:ATP: $\mathrm{Mg}^{2+}$ complex [14]. This explicit solvent, MD free energy approach does not involve any adjustable parameters. As a further test, the same explicit solvent approach was used 
to compute the binding free energy difference between Tc and Mc or doxycycline (Dc) in the primary site, yielding good agreement with experiment (not shown; manuscript in preparation).

\section{Computational methods: Poisson-Boltzmann (PB) binding free energies}

As explained in the main text, several of the binding processes are studied with a simplified, semi-empirical model, which is reasonably well-established, although a number of variants have been used. The processes studied in this way include the competition between Tc binding to TET1 and TET5, as well as the relative stabilities of different Tc tautomers binding to either TET1 or TET5. The electrostatic contribution to the binding free energy is obtained by solving the Poisson-Boltzmann (PB) equation for the bound and unbound forms. This approach has been extensively described in the literature and textbooks; see eg $[8,9,15]$. Importantly, the method does not attempt to compute absolute binding free energies, but only binding free energy differences between two similar ligands (eg, Tc and minocycline), or two putative sites (eg, TET1 and TET5). Because an $\mathrm{Mg}^{2+}$ is already in place in both TET1 and TET5 before Tc: $\mathrm{Mg}^{2+}$ binds, we consider $\mathrm{Mg}^{2+}$ dissociation from the binding site (TET1 or TET5), followed by $\mathrm{Tc}: \mathrm{Mg}^{2+}$ binding to the site. Thus, to compute the (electrostatic contribution to the) binding free energy in the TET1 site, for example, we consider two systems: the ribosome: $\mathrm{Tc}_{\mathrm{Mg}} \mathrm{Mg}^{2+}$ complex along with a free $\mathrm{Mg}^{2+}$ ion; and the ribosome: $\mathrm{Mg}^{2+}$ complex along with a free Tc: $\mathrm{Mg}^{2+}$. Subtracting their electrostatic free energies yields the electrostatic contribution to the binding free energy in the TET1 site. Subtracting the analogous free energy for the TET5 site, we obtain the binding free energy difference between sites.

Previous work with this class of models has sometimes involved the explicit treatment of other, "non-electrostatic" contributions. This is well-illustrated by a recent study of aminoglycosidic antibiotics binding to the 30S ribosome particle by McCammon and coworkers [16]; see their Eq. 1. Additional terms treated there include rotation/translation entropy of the ligand and loss of conformational entropy due to torsional degrees of freedom of the ligands that become ordered upon binding. These terms are not relevant here. The first will largely cancel when TET1 and TET5 are compared, or Tc and Mc. The rigidity of Tc and its fused-ring structure makes the second unimportant. Two other terms considered by McCammon and several other groups are a solvent-accessible surface area term $\left(\Delta G_{n p}\right.$ in Eq. 1 of [16]) and changes 
in the van der Waals interactions between the ligand and its surroundings upon binding $\left(\Delta G_{\text {strain }}\right.$ in Eq. 1 of $[16] ; \Delta G_{v d W}$ in $\left.[17,18]\right)$. These terms are neglected in the present work, for reasons discussed in the following section and in several review articles $[8,15,16,19]$.

The details of the PB calculations are as follows. The electrostatic free energy is obtained by numerically solving the Poisson-Boltzmann equation, using a cubic grid and a finite-difference algorithm implemented in CHARMM [20]. The ionic strength is set to the physiological value of $100 \mathrm{mM}$ (monovalent salt concentration). Doubling this value changes the results only slightly (by $0.3 \mathrm{kcal} / \mathrm{mol}$ for the TET1/TET5 Tc binding free energy difference). Repeating the calculations with a $32 \AA$ radius sphere changes the results negligibly (Tc binding to TET1 changes by $0.1 \mathrm{kcal} / \mathrm{mol}$ ), even though the number of ribosome atoms almost doubles with this larger model. This strongly suggests that the $26 \AA$ model size is reasonable, both for the continuum electrostatic and the MD calculations. The structure of the ribosome:Tc, :Mc, and :Dc complexes are taken from the MD simulations. Calculations are performed for multiple structures, taken every 4 ps along the equilibrated 2.0 ns trajectory, for a total of 250 structures per ligand. The separate ligand and ribosome structures are obtained by simply discarding the unwanted partner. Thus, structural relaxation on separating protein and ligand is not explicitly included (but it is implicit in the dielectric constant). The solvent dielectric constant was set to 80 . The solute dielectric constant was varied from 4 to 16. The data in Table 2 show that Tc binds to both the primary and secondary sites in its zwitterionic form with an associated $\mathrm{Mg}$. The data in Table 3 and 4 show that a dielectric of $6-8$ is optimal and that Tc binding to the secondary site is about 3 $\mathrm{kcal} / \mathrm{mol}$ weaker than the primary site.

\section{Computational methods: neglecting non-electrostatic terms in the Poisson-Boltzmann binding calculations}

Poisson-Boltzmann binding calculations have been used to study a wide variety of protein:ligand complexes $[8,9,15,16,19,21-26]$. One either compares two ligands binding to the same site, or (more rarely) one ligand binding to two sites. In many cases, the so-called MMPB-SA method has been used [23], where van der Waals contributions are explicitly calculated, as well as surface area terms and even vibrational entropy contributions. This poses two problems: (a) these contributions are often noisy, being obtained as differences between two large numbers (eg, the total van der Waals energies of the bound and unbound complexes); (b) the corresponding model 
cannot be derived rigorously, either from thermodynamic perturbation theory or from continuum electrostatics; rather it has a heuristic character; see [15] for a discussion. In other cases $[16,21,22,24,25]$, the focus has been on problems where electrostatic interactions are thought to play a dominant role, so that the continuum electrostatics model is used and other contributions are assumed to cancel. For the present system, where $\mathrm{Tc}^{-}$binding to TET1 and TET5 are compared, four arguments suggest that the $\mathrm{PB}$, electrostatic contribution provides a reasonable approximation to the overall effect. First, the ligand is an anion, binding directly to a $\mathrm{Mg}^{2+}$ cation, close to several ribosome phosphate groups (Fig. 1 in main text). Thus, electrostatic effects should be predominant. Note that this is true not only for the relative binding to TET1 and TET5, but for comparing the neutral and zwitterionic Tc tautomers binding to either site. Second, by adjusting the solute dielectric constant to reproduce binding free energy differences between Tc, Dc, Mc, as well as the $\mathrm{Mg}^{2+}$ binding free energy difference between TET1 and TET5 (see below), we implicitly include other energy terms in the model. In continuum electrostatics, the dielectric constant takes into account the work needed to polarize the medium around a new charge [27]; this work arises partly from elastic deformation of the medium and atomic packing effects mediated by van der Waals and stereochemical energy terms. Notice that both TET1 and TET5 are preorganized for Tc binding: when the MD structures with and without Tc are compared, the rms deviation between the two is just $1.0 \AA$ for both sites and for groups within $7 \AA$ of the ligand. Thus, elastic deformation of either site in response to the ligand is small. Third, we can make a rough estimate of the contribution of van der Waals and accessible surface area effects to the relative binding free energy of Tc to TET1 and TET5, using the Extended Linear Response method of Aqvist, Jorgensen and coworkers; see $[17,18,28]$ for details. The mean van der Waals interaction energy of Tc with its surroundings is computed for Tc bound to TET1 or TET5. These energies are denoted $E_{v d W}^{T E T 1}$ and $E_{v d W}^{T E T 5}$. We emphasize that they represent interactions between Tc and its surroundings, not total van der Waals energies. With the Linear Response method, the van der Waals contribution to the TET1/TET5 binding free energy difference has the form $\Delta G_{v d W}^{T E T 1 / T E T 5}=\alpha\left(E_{v d W}^{T E T 1}-E_{v d W}^{T E T 5}\right)$ (see, eg, Eq. 4 of [17]). Values of $\alpha$ of between 0.10 and 0.40 have been commonly used [17, 18, 28]. For the present system, we obtain $E_{v d W}^{T E T 1}-E_{v d W}^{T E T 5}=-5 \pm 2 \mathrm{kcal} / \mathrm{mol}$. Thus, the Extended Linear Response estimate for $\Delta G_{v d W}^{T E T 1 / T E T 5}$ would be around $-1.0 \pm 0.5 \mathrm{kcal} / \mathrm{mol}$; the negative sign indicates that this contribution favors the TET1 site. Since this contribution is at least partly included in the PB term, the value obtained is likely to be an overestimate. Note that the mean van der Waals interactions between the empty 
TET1 site and its environment, and between the empty TET5 site and its environment, are practically the same. Another term often included in simple binding models is a surface area term, which attempts to capture contributions from the hydrophobic effect. The solvent-accessible surface areas lost by the Tc ligand when it binds to TET1 and TET5 are 715 and $915 \AA^{2}$, respectively. McCammon and coworkers used a surface term in combination with $\mathrm{PB}$ calculations to study aminoglycosidic antibiotics binding to the $30 \mathrm{~S}$ ribosome particle [16]. Using their surface tension of about $5 \mathrm{cal} / \mathrm{mol} / \AA^{2}$, this leads to a $1 \mathrm{kcal} / \mathrm{mol}$ contribution to the TET1/TET5 binding free energy, favoring TET5. This contribution almost exactly cancels the van der Waals estimate above. Both terms are compatible with the estimated uncertainty of our TET1/TET5 comparison (see main text). Both terms are comparable to values obtained for other ligands binding to the $30 \mathrm{~S}$ ribosome particle (see $\Delta G_{\text {strain }}$ and $\Delta G_{n p}$ terms in [16]; see $\Delta G_{v d W}$ term in [29]), both in absolute value and in comparison to the electrostatic term. Finally, the fourth argument suggesting that non-electrostatic contributions are small, is the good agreement between our computed TET1/TET5 binding free energy difference and the observed electron density maps.

\section{Computed occupancies of TET1 and TET5}

$30 \mathrm{~S}$ complexes with 0,1 , or 2 bound Tcs are compared through the thermodynamic cycle in Fig. 1. State 0 (top) corresponds to the unbound partners; states 1 and 1' correspond to Tc bound to either the primary or the secondary site; in state 2, both binding sites are occupied. The partition functions of these states can be written:

$$
\begin{aligned}
& Z_{0}=e^{-\beta G_{0}} \\
& Z_{1}=e^{-\beta G_{1}}+e^{-\beta G_{1}^{\prime}} \\
& Z_{2}=e^{-\beta G_{2}}
\end{aligned}
$$

where $G_{0}, G_{1}, G_{1}^{\prime}, G_{2}$ represent free energies of the corresponding states. Then,

$$
\begin{aligned}
\Delta G_{a} & =-k T \ln \left(Z_{1} / Z_{0}\right) \\
\Delta G_{b} & =-k T \ln \left(Z_{2} / Z_{1}\right)
\end{aligned}
$$

We define $\beta=1 / k T$, where $k$ is Boltzmann's constant and $T$ the temperature; $\alpha=$ $\exp (-\beta \delta \delta G) ; \delta \delta G=\delta G_{1}^{\prime}-\delta G_{1}$ is the binding free energy difference between Tc in the secondary and primary sites. Equations (1) and (2) give:

$$
\Delta G_{b}=\Delta G_{a}+k T(1+\alpha)\left(1+\alpha^{-1}\right)
$$




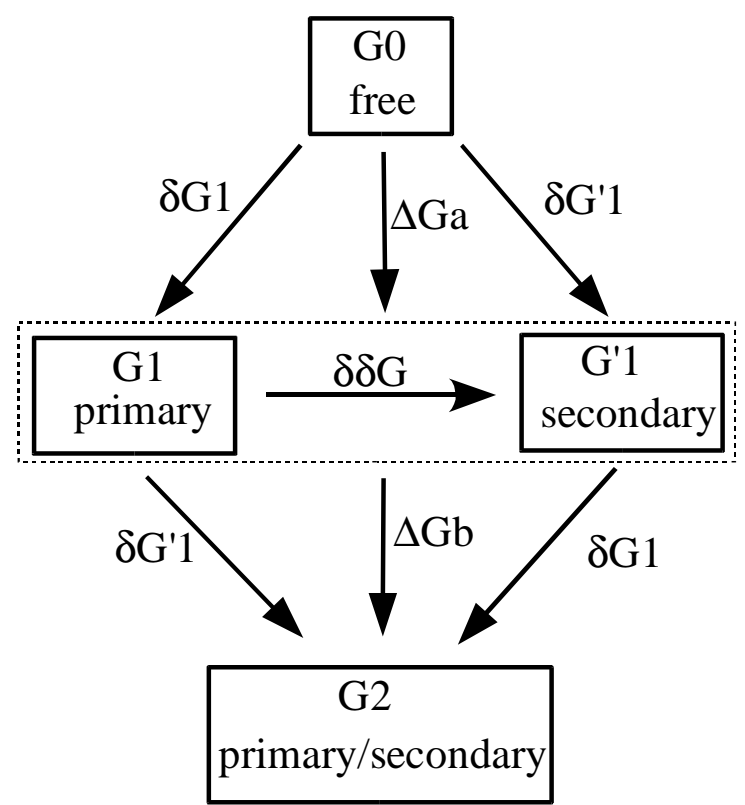

Figure 1: Thermodynamic cycle for Tc:ribosome binding.

At equilibrium:

$$
\begin{aligned}
\Delta G_{a} & =-k T \ln [R: T]_{1} /([R][T]) \\
\Delta G_{b} & =-k T \ln [R: T]_{2} /\left([R: T]_{1}[T]\right)
\end{aligned}
$$

where $[R: T]_{1}$ is the concentration of ribosomes with a single Tc bound (either in the primary or secondary site); $[\mathrm{R}: \mathrm{T}]_{2}$ is the concentration of ribosomes with Tc bound in both sites; $[\mathrm{R}],[\mathrm{T}]$ are the concentrations of free ribosomes and tetracyclines. The total concentrations of ribosomes and tetracyclines are:

$$
[R]_{\text {tot }}=[R: T]_{1}+[R: T]_{2}+[R]
$$




$$
[T]_{t o t}=[R: T]_{1}+2[R: T]_{2}+[T]
$$

The association constant is:

$$
K_{a}=[R: T]_{1} /([R][T])
$$

Together, (3-7) form a closed system of equations. For the concentration of complexes with Tc bound to the primary site, we obtain:

$$
[R: T]_{1}^{\mathrm{TET} 1}=[R: T]_{1} /(1+\alpha)
$$

For the ratio of complexes with Tc in TET5 and TET1, we obtain:

$$
\frac{[T c]_{\mathrm{TET} 5}}{[T c]_{\mathrm{TET} 1}}=\frac{[R: T]_{2}}{[R: T]_{1}^{\mathrm{TET} 1}+[R: T]_{2}}
$$

The experimental total concentration of ribosomes in the crystal [1] was estimated from the volume of the unit cell as $5010^{-6} \mathrm{M}$; the total concentration of tetracyclines was $8010^{-6} \mathrm{M}$. The association constant $\mathrm{K}_{a}$ is thought to be in the range $0.110^{6} \mathrm{M}^{-1}$ to $1.010^{6} \mathrm{M}^{-1}[30-32]$.

\section{Results: $\mathrm{Mg}^{2+}$ is pre-bound in TET1 and TET5}

By reversibly deleting $\mathrm{Mg}^{2+}$ from either TET1, TET5, or from bulk water, we can show that $\mathrm{Mg}^{2+}$ is strongly bound in both sites in the absence of any ligand. MD simulations are done with explicit solvent and the free energy change is obtained by thermodynamic integration (see above). The data are given in Table 1.

Table 1: Free energy to delete $\mathrm{Mg}^{2+}$ cations from the primary and secondary ribosomal binding sites using MD with explicit solvent

\begin{tabular}{ccc}
\hline Medium & $\Delta G\left(\mathrm{Mg}^{2+} \rightarrow\right.$ nothing $)$ & $\Delta \Delta G$ \\
\hline solution & $440^{a}$ & - \\
TET1 site & $465.9 / 469.0$ & 27.5 \\
TET5 site & $454.5 / 450.6$ & 12.5 \\
\hline
\end{tabular}

Energies are in $\mathrm{kcal} / \mathrm{mol}$. ${ }^{a}$ Solution value is for $\mathrm{Mg}^{2+}$ in pure water, from a previous study [33]. For TET1 and TET5, two simulations were done, giving two free energy results. $\Delta \Delta G$ is the difference between $\mathrm{Mg}^{2+}$ deletion in the ribosome and in pure water. A positive value indicates a favorable binding to the ribosome site. 


\section{Results: Tc binds in the zwitterionic form to TET1 and TET5}

The Poisson-Boltzmann free energy results are in Table 2. The zwitterionic state is preferred in solution [21]. Its binding free energies are lower than those of the neutral form, so that it is the predominant form in both sites.

Table 2: Poisson-Boltzmann free energy simulations comparing the binding of $\mathrm{Tc}^{-}: \mathrm{Mg}^{2+}$ to the primary and secondary site. Energies are in kcal/mol.

\begin{tabular}{ccccccc}
\hline binding site & Tc form & $\mathrm{Mg}^{2+}$ & \multicolumn{5}{c}{$\epsilon$, dielectric constant } \\
& & & 4 & 6 & 8 & 12 \\
\hline primary & neutral & yes & -54.0 & -39.4 & -31.6 & -23.5 \\
primary & zwitterionic & yes & -78.2 & -55.8 & -44.0 & -31.8 \\
secondary & neutral & yes & -25.1 & -19.5 & -16.4 & -13.3 \\
secondary & zwitterionic & yes & -40.5 & -30.2 & -24.7 & -19.0 \\
\hline
\end{tabular}

\section{Results: Calibrating the dielectric constant to match Dc and Mc binding}

We may assume the ligands all bind mainly to the primary site in their zwitterionic forms with $\mathrm{Mg}$ present. For Mc, the experimental binding free energy difference is $-2.6 \mathrm{kcal} / \mathrm{mol}$, favoring Mc over Tc. Since Dc has the same biological activity as Tc, we assume their binding free energies are equal. These experimental values are best reproduced with a dielectric constant of 6 or 8 (Table 3 ). 
Table 3: Poisson-Boltzmann free energy simulations comparing binding of zwitterionic tetracycline $(\mathrm{Tc})$, minocycline $(\mathrm{Mc})$ and doxycycline $(\mathrm{Dc})$ to the TET1 site.

\begin{tabular}{|c|c|c|c|c|c|}
\hline \multirow[t]{2}{*}{ ligand } & \multicolumn{4}{|c|}{$\epsilon$, dielectric constant } & \multirow[t]{2}{*}{$\Delta \Delta G_{\text {expt }}$} \\
\hline & 4 & 6 & 8 & 12 & \\
\hline $\mathrm{Tc}^{-}: \mathrm{Mg}^{2+}$ & -78.2 & -55.8 & -44.0 & -31.8 & \\
\hline $\mathrm{Mc}^{-}: \mathrm{Mg}^{2+}$ & -81.1 & -57.8 & -45.1 & -32.9 & \\
\hline$\Delta \Delta G(M c \rightarrow T c)$ & $-2.9(2.0)$ & $-2.0(1.3)$ & $-1.5(1.0)$ & $-1.1(0.8)$ & -2.6 \\
\hline $\mathrm{Dc}^{-}: \mathrm{Mg}^{2+}$ & -76.6 & -54.7 & -43.2 & -31.4 & \\
\hline$\Delta \Delta G(D c \rightarrow T c)$ & $1.6(2.0)$ & $1.1(1.3)$ & $0.8(1.0)$ & $0.4(0.8)$ & 0.0 \\
\hline
\end{tabular}

Poisson-Boltzmann free energies, in $\mathrm{kcal} / \mathrm{mol}$. Values correspond to the binding of $\mathrm{Tc}^{-}: \mathrm{Mg}^{2+}, \mathrm{Dc}^{-}: \mathrm{Mg}^{2+}$, and $\mathrm{Mc}^{-}: \mathrm{Mg}^{2+}$ to the primary site. Negative $\Delta \Delta G(M c \rightarrow T c)$ values correspond to stronger Mc binding. The experimental result for Mc is $-2.6 \mathrm{kcal} / \mathrm{mol}$ [30] and $0.0 \mathrm{kcal} / \mathrm{mol}$ for Dc [34].

\section{Results: Tc binds preferentially to the primary, TET1 site}

We use Poisson-Boltzmann free energy calculations. We follow a protocol that compares the binding of $\mathrm{Mg}^{2+}$ alone, and the binding of $\mathrm{Tc}^{-}: \mathrm{Mg}^{2+}$. This has the advantage that $\mathrm{Tc}$ is always associated with $\mathrm{Mg}^{2+}$, and the large energy associated with $\mathrm{Tc}^{-}: \mathrm{Mg}^{2+}$ separation never appears explicitly. When the two sites and ligands are compared, the contributions due to $\mathrm{Mg}^{2+}$ cancel, as they should. Thus, the line "secondary - primary" in Table 4 gives the Tc binding free energy difference $\delta \delta G$ between sites. For all values of the solute dielectric constant, $\delta \delta G$ is negative, ie, the primary site is favored. Individul group contributions to TET1 binding are shown in Table 5. The phosphate groups of Cyt1054, Gua1197, and Gua1198 make the largest contributions by far. 
Table 4: $\mathrm{Tc}^{-}: \mathrm{Mg}^{2+}$ and $\mathrm{Mg}^{2+}$ binding to the primary and secondary sites.

\begin{tabular}{cccccc}
\hline binding site & ligand & \multicolumn{4}{c}{$\epsilon$, dielectric constant } \\
& & 4 & 6 & 8 & 12 \\
\hline primary & $\mathrm{Mg}^{2+}$ & -95.5 & -71.2 & -58.3 & -44.7 \\
secondary & $\mathrm{Mg}^{2+}$ & -59.9 & -48.5 & -42.1 & -34.8 \\
difference & $\mathrm{Mg}^{2+}$ & -35.6 & -22.7 & -16.2 & -9.9 \\
primary & $\mathrm{Tc}^{-}: \mathrm{Mg}^{2+}$ & -78.2 & -55.8 & -44.0 & -31.8 \\
secondary & $\mathrm{Tc}^{-}: \mathrm{Mg}^{2+}$ & -40.5 & -30.2 & -24.8 & -19.0 \\
difference & $\mathrm{Tc}^{-}: \mathrm{Mg}^{2+}$ & -37.7 & -25.6 & -19.2 & -12.8 \\
secondary-primary & & $2.1(2.0)$ & $2.9(2.0)$ & $3.0(2.0)$ & $2.9(2.0)$ \\
\hline
\end{tabular}

Poisson-Boltzmann free energies, in $\mathrm{kcal} / \mathrm{mol}$. Values correspond to the binding of $\mathrm{Mg}^{2+}$ or $\mathrm{Tc}^{-}: \mathrm{Mg}^{2+}$ to the primary or secondary site. Tc was deleted from the pocket when $\mathrm{Mg} 2+$ binding was computed. Positive secondary-primary values correspond to preferential Tc binding to the primary site.

Table 5: Contributions $(\mathrm{kcal} / \mathrm{mol})$ of individual groups to the Poisson-Boltzmann free energy of binding $\mathrm{Tc}^{-}: \mathrm{Mg}^{2+}$ to the TET1 site; dielectric constant $\epsilon=8$

\begin{tabular}{cccccc}
\hline nucleotide & backbone & base & nucleotide & backbone & base \\
\hline Gua1197 & -0.0 & -5.6 & Gua963 & 0.0 & -0.1 \\
Cyt1054 & -0.9 & -4.6 & Ura1052 & 0.0 & -0.1 \\
Gua1198 & 0.0 & -5.0 & Ade1055 & 0.0 & 0.0 \\
Gua1053 & -0.0 & -1.1 & Cyt967 & -0.2 & 0.2 \\
Ura1199 & 0.1 & -1.0 & Ura1194 & 0.0 & 0.0 \\
Ura1196 & -0.1 & -0.6 & Cyt1195 & -0.7 & 0.8 \\
Ade964 & 0.0 & -0.3 & Ade968 & 0.0 & 0.1 \\
Ade965 & 0.0 & -0.2 & Gua966 & 0.0 & 1.0 \\
\hline
\end{tabular}

\section{References}

[1] Brodersen, D. E., Clemons Jr., W. M., Carter, A. P., MorganWarren, R. J., Wimberly, B. T., and Ramakrishnan, V. The structural 
basis for the action of the antibiotics tetracycline, pactamycin, and hygromycin B on the 30S ribosomal subunit. Cell 103 (2000), 1143-154.

[2] Nina, M., And Simonson, T. Molecular dynamics of the tRNA ${ }^{\text {Ala }}$ acceptor stem: comparison between continuum reaction field and particle-mesh Ewald electrostatic treatments. J. Phys. Chem. B 106 (2002), 3696-3705.

[3] Beglov, D., And Roux, B. Finite representation of an infinite bulk system: solvent boundary potential for computer simulations. J. Chem. Phys. 100 (1994), 9050-9063.

[4] Mackerell, A., Bashford, D., Bellott, M., Dunbrack, R., Evanseck, J., Field, M., Fischer, S., Gao, J., Guo, H., Ha, S., Joseph, D., KuchNir, L., Kuczera, K., Lau, F., Mattos, C., Michnick, S., Ngo, T., Nguyen, D., Prodhom, B., Reiher, W., Roux, B., Smith, J., Stote, R., Straub, J., Watanabe, M., Wiorkiewicz-Kuczera, J., Yin, D., And KARPLus, M. An all-atom empirical potential for molecular modelling and dynamics study of proteins. J. Phys. Chem. B 102 (1998), 3586-3616.

[5] Jorgensen, W., Chandrasekar, J., Madura, J., Impey, R., and Klein, M. Comparison of simple potential functions for simulating liquid water. J. Chem. Phys. 79 (1983), 926-935.

[6] Stote, R., States, D., and Karplus, M. On the treatment of electrostatic interactions in biomolecular simulation. J. Chim. Phys. 88 (1991), 2419-2433.

[7] Aleksandrov, A., And Simonson, T. The tetracycline: $\mathrm{Mg}^{2+}$ complex: a molecular mechanics force field. J. Comp. Chem. 13 (2006), 1517-1533.

[8] Simonson, T., Archontis, G., And Karplus, M. Free energy simulations come of age: the protein-ligand recognition problem. Acc. Chem. Res. 35 (2002), 430-437.

[9] McCammon, J. Computation of noncovalent binding affinities. In Theory and Applications of Computational Chemistry, C. Dykstra, G. Frenking, K. Kim, and G. Scuseria, Eds. Elsevier, Amsterdam, 2005, pp. 41-46.

[10] Brooks, B., Bruccoleri, R., Olafson, B., States, D., Swaminathan, S., AND KARPlus, M. Charmm: a program for macromolecular energy, minimization, and molecular dynamics calculations. J. Comp. Chem. 4 (1983), 187-217. 
[11] Kollman, P. Free energy calculations: applications to chemical and biochemical phenomena. Chem. Rev. 93 (1993), 2395.

[12] Simonson, T. Free energy calculations. In Computational Biochemistry \& Biophysics, O. Becker, A. Mackerell Jr., B. Roux, and M. Watanabe, Eds. Marcel Dekker, N.Y., 2001, ch. 9.

[13] Jorgensen, W., Buckner, K., Boudon, S., and Tirado-Rives, J. Efficient computation of absolute free energies of binding by computer simulations. Application to the methane dimer in water. J. Chem. Phys. 89 (1988), 3742-3746.

[14] Thompson, D., And Simonson, T. Molecular dynamics simulations show that bound $\mathrm{mg}^{2+}$ contributes to amino acid and aminoacyl adenylate binding specificity in aspartyl-trna synthetase through long range electrostatic interactions. J. Biol. Chem. 281 (2006), 23792-23803.

[15] Simonson, T. Free energy calculations: approximate methods for biological macromolecules. In Free energy calculations: theory and applications in chemistry and biology, C. Chipot and A. Pohorille, Eds. Springer Verlag, N.Y., 2006, ch. 12.

[16] Yang, G., Trylska, J., Tor, Y., and McCammon, J. A. Binding of aminoglycosidic antibiotics to the oligonucleotide A-site model and 30S ribosomal subunit: Poisson-boltzmann model, thermal denaturation, and fluorescence studies. J. Med. Chem. 49 (2006), 5478-5490.

[17] Ostrovsky, D., Udier-Blagovic, M., And Jorgensen, W. Analyses of activity for factor $\mathrm{X} \alpha$ inhibitors based on Monte Carlo simulations. J. Med. Chem. 46 (2003), 5691-5699.

[18] Kroeger-Smith, M., Hose, B., Hawkins, A., Lipchock, J., Farnsworth, D., Rizzo, R., Tirado-Rives, J., Arnold, E., Zhang, W., Hughes, S., Jorgensen, W., Michedja, C., And Smith, R. Molecular modeling calculations of HIV-1 reverse transcriptase nonnucleoside inhibitors: correlation of binding energy with biological activity for novel 2-aryl-substituted benzimidazole analogues. J. Med. Chem. 46 (2003), 1940-1947.

[19] McDowell, S. E., Spackova, N., Sponer, J., And Walter, N. G. Molecular dynamics simulations of RNA: An in silico single molecule approach. Biopolymers 85 (2007), 169-184. 
[20] Im, W., Beglov, D., ANd Roux, B. Continuum solvation model: computation of electrostatic forces from numerical solutions to the Poisson-Boltzmann equation. Comp. Phys. Comm. 111 (1998), 59-75.

[21] Aleksandrov, A., Proft, J., Hinrichs, W., and Simonson, T. Protonation patterns in tetracycline:tet repressor recognition: Simulations and experiments. ChemBioChem 8 (2007), 675-685.

[22] Hendsch, Z., And Tidor, B. Electrostatic interactions in the GCN4 leucine zipper: substantial contributions arise from intramolecular interactions enhanced on binding. Prot. Sci. 8 (1999), 1381-1392.

[23] Kollman, P., Massova, I., Reyes, C., Kuhn, B., Huo, S., Chong, L., Lee, M., Lee, T., Duan, Y., Wang, W., Donini, O., Cieplak, P., Srinivasan, J., Case, D., And Cheatham, T. Calculating structures and free energies of complex molecules: combining molecular mechanics and continuum models. Acc. Chem. Res. 33 (2000), 889-897.

[24] Archontis, G., Simonson, T., And Karplus, M. Binding free energies and free energy components from molecular dynamics and Poisson-Boltzmann calculations. Application to amino acid recognition by aspartyl-tRNA synthetase. J. Mol. Biol. 306 (2001), 307-327.

[25] Thompson, D., Lazennec, C., Plateau, P., and Simonson, T. Ammonium scanning in an enzyme active site: the chiral specificity of aspartyl-tRNA synthetase. J. Biol. Chem. in press (2007), 0000.

[26] Wong, C., Hunenberger, P., Akamine, P., Narayana, N., Diller, T., McCammon, J., Taylor, S., And Xuong, N. Computational analysis of PKA-balanol interactions. J. Med. Chem. 44 (2001), 1530-1539.

[27] FrÖHLICH, H. Theory of Dielectrics. Clarendon Press, Oxford, 1949.

[28] Aqvist, J., Luzhkov, V., and Brandsal, B. Ligand binding affinities from MD simulations. Acc. Chem. Res. 35 (2002), 358-365.

[29] Almlöf, M., Ander, M., And Aqvist, J. Energies of codon-anticodon recognition on the small ribosomal subunit. Biochemistry 46 (2007), 200-209. 
[30] Olson, M. W., Ruzin, A., Feyfant, E., Rush, T. S., O'Connell, J., And BRADFORD, P. A. Functional, biophysical, and structural bases for antibacterial activity of tigecycline. Antimicrob. Agents Chemoth. 50 (2006), 2156-2166.

[31] Wissmann, A., And Hillen, W. Forum Mikrobiol. (1989), 292-299.

[32] Epe, B., And Wolley, P. EMBO J. 3 (1984), 121-126.

[33] Thompson, D., Plateau, P., and Simonson, T. Free energy simulations reveal long-range electrostatic interactions and substrate-assisted specificity in an aminoacyl-tRNA synthetase. ChemBioChem 7 (2006), 337-344.

[34] Ross, J., Eady, E., Cove, J., and Cunliffe, W. Antimicrob. Agents Chemoth. 42 (1998), 1702-1705. 